\title{
Sea Otter Carrying Capacity in a Soft- and Mixed-sediment Benthic Habitat
}

\author{
Ian Davis ${ }^{1}$, Timpthy Dellapenna ${ }^{1}$, Gary Maale $^{2}$, Francis Gelwick ${ }^{2}$, Fred Selts ${ }^{3}$, and Randall \\ Davis $^{4}$
}

${ }^{1}$ Texas A\&M University College Station

${ }^{2}$ Texas A and M University College Station

${ }^{3}$ None

${ }^{4}$ Texas University at Galveston

January 6, 2021

\begin{abstract}
Identifying factors that influence sea otter (Enhydra lutris) population density can provide insight into why it varies spatially and temporally and when a recovering population has reached an equilibrium density because of food resources (i.e., carrying capacity K). Although food availability is widely recognized as an important extrinsic factor affecting sea otter density, how do we determine when a population has reached K? The goal of this study was to estimate K for Simpson Bay, Alaska by measuring the abundance of edible bivalves, the primary prey for sea otters for over 40 years. We then compared prey abundance and estimated replacement rate (i.e., the mean age of bivalves predated by sea otters) to estimated annual prey consumption based on the mean population density for the past 18 years. On average, 110 adult sea otters $(5.2 \mathrm{~km}-2)$ have occupied Simpson Bay annually since 2001 consuming an estimated $176,660 \mathrm{~kg}$ of bivalves. The total mass (standing stock) of the major bivalves (predominately butter clams and stained macomas) was $785,730 \mathrm{~kg}$, so adult sea otters consumed about $22 \%$ annually. Based on these observations and calculations, the estimated annual number of sea otters occupying Simpson Bay appears to be at or near $\mathrm{K}$ based on the replacement rate of food resources. However, other intrinsic (e.g., male territoriality and emigration) and extrinsic (e.g., predation, disease, human-related mortality) factors may influence equilibrium density, which varies spatially and temporally, resulting in a mosaic of subpopulations with different densities, rates of growth and discontinuous distributions. Understanding the balance among these factors may be one of the most challenging ecological questions for sea otter conservation and management as populations recover from their range-wide decimation during the Maritime Fur Trade in the late 18th and 19 th centuries
\end{abstract}

\section{Hosted file}

Davis et al. Sea otter carrrying capacity.pdf available at https://authorea.com/users/387825/ articles/502749-sea-otter-carrying-capacity-in-a-soft-and-mixed-sediment-benthic-habitat 